\title{
Automatic Quantitative Measurements In SEM
}

\author{
Sergey Babin
}

Abeam Technologies, Inc.

5286 Dunnigan Ct., Castro Valley, CA 94546, USA; sb@abeamtech.com

Quantitative measurements at the nanoscale are an enabler of progress in nanosciences and nanofabrication. Accurate measurements of critical dimensions (CD) in a scanning electron microscope (SEM) is a complex task [1]. Methods of extracting this information based purely on the analysis of image brightness could yield results far from the actual CD values. Indeed, extraction of the brightest spot or the largest derivative at the edge of a line is often used, which do not represent the edge of a line; neither at the top nor bottom. Furthermore, the measurement error depends on many factors such as the materials, beam size, and accelerating voltage. So, the CD values calibrated for a specific pattern or SEM setup cannot be applied to the analysis of other patterns or images received in slightly different conditions of observation.

Study of electron interaction with a 3D sample composed of various materials is the key to understanding signal formation in SEM. Special attention was given to the modeling of slow energy secondary electrons: they contribute a significant part to the SEM signal and they are sensitive to a variation in materials, geometry of a sample, and to detection track. Advanced models of electron scattering were used to analyze secondary emission from a sample: elastic and inelastic electron scattering, generation of fast secondary electrons, plasmon mechanisms, electron propagation between layers, and generation of true secondary electrons.

Electron travel in the presence of an electromagnetic field was also modeled. It is important to simulate the trajectories as well as the electron detector, its geometry, placement, and energy transfer function. As a result, a comprehensive model of SEM enabled an accurate prediction of SEM signal.

Extensive simulations helped us to understand the signal formation and its relationship to a variety of parameters involved with the formation of the SEM image: beam voltage, materials, wall angle, distance to the neighboring feature, layer thickness, set-up of detectors, etc.

Based on a physical model, the myCD software was developed to analyze an SEM image and extract information regarding linewidth, line width roughness, and the wall angle of features. Some parameters, such as the beam voltage and materials, should be known to the operator as the input data, along with the SEM image. Using this information, the software builds a model and extracts contours of the features at the top and bottom.

One of the critical parameters which affects measurement results in SEM is the electron beam size. The knife edge method is often used, which is inaccurate and also operator dependent. Automatic measurement of the beam size was developed. A spatial frequency analysis was used to extract the beam size out of a known, specially fabricated sample. ${ }^{2}$ 


\section{References}

1. D. C. Joy, Proc. SPIE, 3332 (1998) 102; also V. Ukraintsev, Proc. SPIE, 5038 (2003) 644

2. S. Babin, C. Peroz, et. al., Microelectronic Engineering, 86 (2009) 524

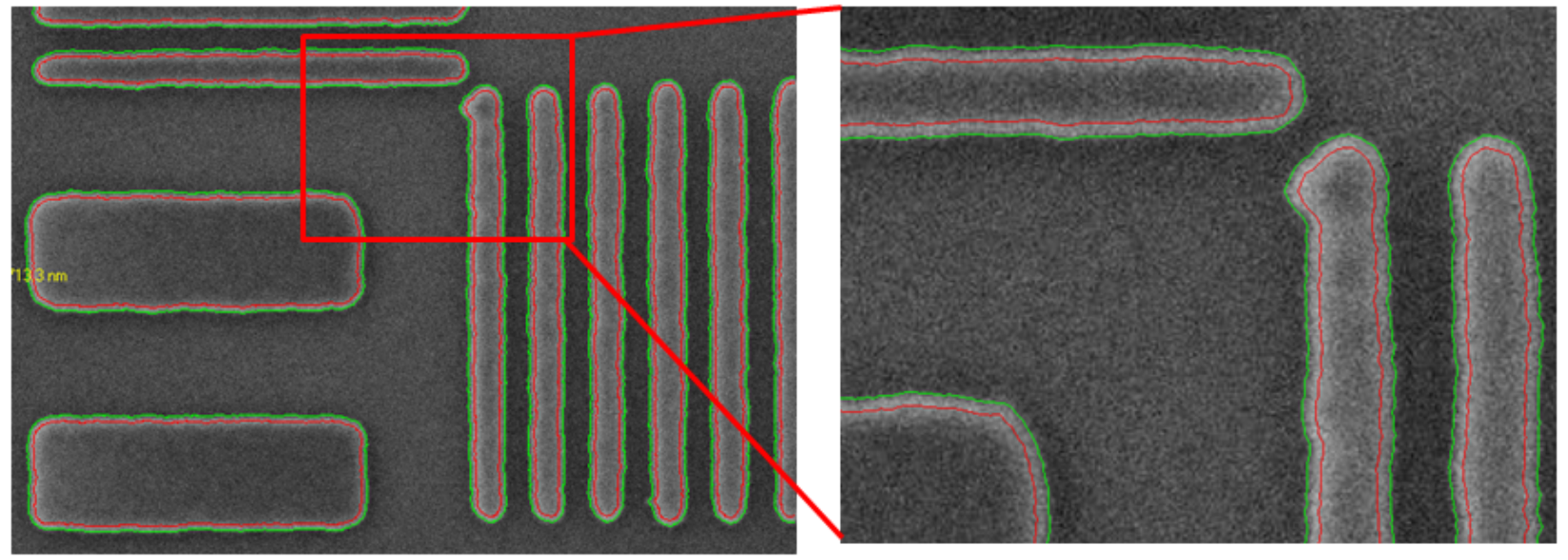

FIG. 1. SEM image with extracted contours at the top and bottom of features. This extraction is done automatically based on the physical model of image formation in SEM. Using these contours, the linewidth at the top, bottom, and 50\% height, as well as line width roughness and sidewall angles, are determined.

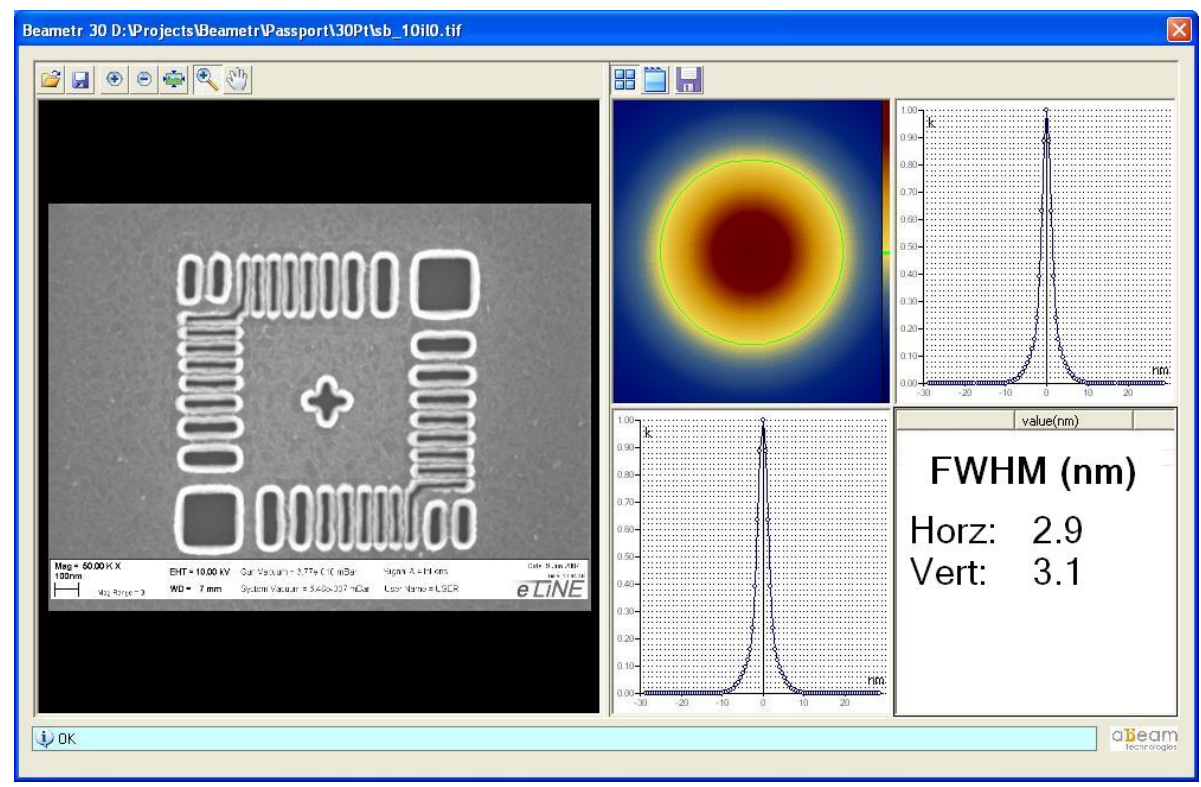

FIG. 2. Knowledge of beam size is critical for dimensional measurements in SEM. A technique for automatic measurement of e-beam size was developed. An SEM image of a specific test sample is shown on the left; results of the beam size extraction are on the right. 